\title{
Human emotion recognition based on block patterns of image and wavelet transform
}

\author{
Pravin B Chopade ${ }^{1 *}$ and Prabhakar N Kota ${ }^{2}$ \\ Associate Professor, Department of Electronics \& Telecommunication Engineering, Modern Education Society's \\ College of Engineering, Pune, India ${ }^{1}$ \\ Assistant Professor, Department of Electronics \& Telecommunication Engineering, Modern Education Society's \\ College of Engineering, Pune, India ${ }^{2}$
}

Received: 31-May-2021; Revised: 01-October-2021; Accepted: 04-October-2021

C2021 Pravin B Chopade and Prabhakar N. This is an open access article distributed under the Creative Commons Attribution (CC BY) License, which permits unrestricted use, distribution, and reproduction in any medium, provided the original work is properly cited.

\begin{abstract}
In this manuscript, Human Emotion Recognition based on Block Patterns of Image and Discrete Wavelet Transform (HER-BP-DWT) is proposed. The different facial parts, such as eyebrows, eye, lips, mouth, and muscle movements play an important role in emotion recognition. But according to change in age, the movements of facial parts and muscles become weaker, so recognizing emotions can be a little more tedious and complicated. Therefore, in this manuscript, a new approach that is different from the conventional one using block patterns and discrete wavelet transform is proposed. Here, first of all, the test image is divided horizontally and vertically into different block patterns. Then, each block is separated as sub blocks. The particular area block is decomposed into different frequency sub bands with the help of discrete wavelet transform. The energy of these sub bands of each block is calculated. The energy of sub bands of the test image and the reference image is compared. The main aim of this proposed method is to recognize emotional expressions using a simple parameter, like energy of sub bands that is obtained from discrete wavelet transform and it is easy to use. The main objective is to increase the accuracy during face image recognition. The proposed HER-BP-DWT method can be efficiently and accurately recognized different emotions, such as happiness, sadness, anger, etc. The proposed method is very convenient to use due to the use of block patterns. The proposed approach is activated in MATLAB platform, then the performance is compared with other existing approaches, such as Human Emotion Recognition using Convolutional Neural Networks (HER-CNN) and Human Emotion Recognition using Bimodal Fusion Algorithm (HER-BFA). Finally, the experimental results show that the HER-BP-DWT method is superior to the existing methods. From the experimental analysis, the HER-BP-DWT method shows the accuracy of $99.55 \%$, sensitivity of $85.93 \%$, precision of $92.43 \%$ and $\mathbf{9 0 . 7 4 \%}$ of specificity, which is prominent than the existing methods.
\end{abstract}

\section{Keywords}

Energy component, Discrete wavelet transform, Image blocks, Pattern, Coefficients of frequency sub bands, Facial emotion expression.

\section{Introduction}

Nowadays, the facial images, such as facial expression, gestures, and other factors are making them hard to recognize accurately. The main issue of facial recognition is "how to remove such harmful factors?" To extract the facial feature, the facial features need some robustness for the beyond factors [1]. Human being normally communicated, either verbally or non-verbally with the world. In both ways of communication, the expression of the face varies with the state of situation [2].

*Author for correspondence
Expression of people is considered as a non-verbal communication. While expressing themselves, words and gestures are not enough to exhibit, but emotion plays a vital role to understand the person's desires or expectations. Through emotions, human can fully express themselves [3]. Therefore, this is a significant way of nonverbal communication between the human. In various application fields, such as robotics, surveillance, information security, human machine interaction, and video editing, the different emotion recognition techniques and its analysis carry enormous significance to run that particular application smoothly [4]. The development of an effective emotional recognition system is still a major 
challenge due to the moment of the muscles under the face skin and the micro expression that is present in the face for a few milliseconds [5, 6]. The facial expression is always the main focus when interacting with each other [7]. These expressions provide a lot of details of social information [8-10]. Furthermore, these expressions may be voluntarily or involuntarily adopted by human, while these are utterly controlled by the nervous system of the human and it differs from person to person [11-13]. From these facial expressions of human, one can easily understand the mental, emotional, and physical state of communication. The emotion recognition system has a wide range of utilizations, viz emotional robot, performance forecasting user-friendly interface amid the man and machine [14-16]. Nevertheless, emotion recognition by untrained person is difficult. Hence, it is significant to know the mind status from expression, because mind status can be easily read from different parts of the face such as mouth, eyes, eyebrows, etc. Proper feature extraction is a noteworthy step of automatic recognition, emotion system [17, 18]. In many self-assessment applications, emotions are being utilized for the improvement of individual as well as company performance, but humans are very skilful in hiding and suppressing emotions. Hence, there is a need to develop a defective and easy emotion recognition technique that can be user friendly and it can be easily implemented in the hardware platform.

The dimension of the higher dimension image information should be minimized, as there is a higher dimension with unnecessary information present in the original facial image. Nevertheless, facial information contains only a smaller portion of fullface information and traditional Principal Component Analysis (PCA) exposure can lead to more information loss [19]. Lastly, classified work can be done through machine learning methods, like Support Vector Machine (SVM) classification, Neural Network (NN). The success of the facial recognition system is to deal with the problem that light, colour and background are exposed to many variations of the complex human face. Therefore, this manuscript proposes the recognition of automated face identification, face expression utilizing wavelet methods under complex backgrounds. This Discrete Wavelet Transform (DWT) method provides high accuracy, high speed with less computational complexity compared with the existing methods.

One on a challenging research topic is human face recognition method. The Facial Expression
Recognition (FER) approaches contain Gabor Wavelet Transform (GWT), Active Appearance Model (AAM), Local Binary Pattern (LBP). The GWT technique extracted face imagery texture information in several scales with direction attains better outcomes [20]. But the cost of time and the complexity of space are high. The facial expression dynamics can capture through analyzing locations of signs from AAM, which is utilized for extracting shape feature. Even though AAM can effectively get facial feature points, the fitting approach has a nonlinear optimized issue, which has increased the calculation process to a more complex as well as computational complexity.

In this manuscript, an emotion recognition algorithm is proposed based on specific block patterns and discrete wavelet transforms. Emotion recognition is carried out by the analysis of the movement of facial muscles, which is very critical for the micro expression and remains very short time on the face. Many researchers have identified the human emotion based on different psychological signals, such as heart rate, brain signal, and skin temperature but recently brain signal has captured a lot of popularity for the recognition of emotion. For the extraction of features either for processing and classification of emotions from the human face, many researchers have adopted different tools ranging from frequency transform into different kinds of wavelet transform along with advanced methods. Several works were presented in the literature to overcome the limitations, such as diminish the dimensionality of face image, loss of information due to lack of space, poor accuracy of the system, but the presented works are not much effective. These drawbacks have motivated to do this research work.

The objective of this proposed algorithm is to identify the emotional expressions with the help of a simple parameter, like energy of sub bands that is obtained from DWT and it is easy to use and it is to increase the accuracy during the face image recognition.

The choice of wavelet transform is driven through its insensitivity. Here, experiments utilized contact as well as start values to ensure greater consistency of the produced classified results. The encouraging test outcomes established the proposed approach utilizing frontal and side-view images are feasible, effective solution to identifying faces, which can lead to, well, practical use of forensic databases that exist in computerized human facial recognition requests. 
The main contributions of this manuscript summarizing below,

1) In this manuscript, human emotion recognition depending on block patterns and DWT is proposed

2) Initially, the test image is divided horizontally and vertically into different block patterns [21].

3) Each block is separated as sub blocks. The particular area block is decomposed into different frequency sub bands with the help of DWT. The energy of these sub bands of each block is calculated [22].

4) The main aim of this proposed algorithm is to identify emotional expressions with the help of a simple parameter, like energy of sub bands that is obtained from DWT and it is easy to use.

5) The proposed algorithm can be able to identify the different emotions, such as happiness, sadness, anger, etc. efficiently and correctly

6) Due to the use of block patterns, the proposed algorithms become convenient to use.

7) The proposed Human Emotion Recognition based on Block Patterns of Image and Discrete Wavelet Transform (HER-BP-DWT) method shows better than the existing methods, such as Human Emotion Recognition using Convolutional Neural Networks (HER-CNN) and Human Emotion Recognition using Bimodal Fusion Algorithm (HER-BFA).

In this work, an explanation of the system description and methodology consists of three parts: block pattern of the image (text/reference image), the use of DWT, and extraction of features and its comparisons have been discussed thoroughly. The rest of this manuscript is structured as follows: section 2 presents the literature review. Section 3 illustrates about the proposed method. Section 4 demonstrates the experimental results and discussions. Section 5 concludes the manuscript.

\section{Literature review}

$\mathrm{Xu}$ et al. [23] have presented the emotional computing in the field of Internet of things (IoT). Here, emotions were identified and classified by feature extraction with the help of double tree complex wavelet transform. The presented algorithm was proved its effectiveness for the extraction of feature vector and it was improved the efficiency of emotion recognition system.

Acharya et al. [24] have presented the combined emotions and brain signal, which was obtained while stimulating the emotions of humans using different movie clips. Furthermore, these brain signals as electroencephalogram device were removed with fast Fourier transform and apply that genetic programming for the classification of emotions.

Zang et al. [25] have introduced bi-orthogonal wavelet entropy for the extraction of multiple scale characteristics with the fuzzy support vector machine was used for classifying emotions from the facial images.

Ayyavoo and Suseela [26] have suggested DWT to minimize the illumination effect of facial images before processing for emotion recognition.

Krishna et al. [27] have presented a tunable Qwavelet transform by utilizing brain signals for the classification of emotions. Here, wavelet transforms decompose the brain signal that was obtained from the electroencephalogram and different features were extracted and processed for classification.

Meena et al. [28] have introduced the facial expression of the input image into local patterns and graph wavelet transform was applied to it to extract the features.

Ekman and Friesen [29] have introduced a legendarily FER named 'Facial Action coding system (FACS)' which was mostly referred by many upcoming researchers and scientists. Authors have discussed different facial emotions such as amazement, fright, anger, hatred, pleasure, sorrow; such emotions were typically utilized in the field of human recognition techniques [30]. The illumination conditions could play a vital role in determining the different emotions from facial images and videos as it affects more in those images.

Sown [31] have suggested the pioneer of the automatic FER method. The suggested system was deemed with the help of different twenty image structures. The suggested system was classified into three different parts: face identification with monitoring, feature extraction, emotion categorization. The features were extracted from the geometry or appearance of facial images or videos whose emotions need to be identified. Skin texture variations, via wrinkles, furrows were studied meticulously in the appearance-based feature extraction system or techniques for whole face including selected face areas.

Li et al. [32] have presented a texture descriptorbased Gabor wavelets were used in plane image 
transform. Most of the comprehensive literature surveys of facial emotion recognition techniques were adopted [33]. The most relevant part of this technique was to remove valid features in either spatial nor Frequency Domain (FD). The count of approaches depending on FD features was Fast Fourier Transform (FFT), Discrete Cosine Transform (DCT), DWT, Discrete Fourier Transform (DFT), GWT, Curve let transform. Presently, different facial elements such eyes, lips, eyebrows, etc., and movement of muscles which remain on a very short duration have not given enough attention on the FER systems field in the FD.

Huang et al. [34] have presented a strong face detected process depends on the skin color enhancement along with FER approach using block PCA. Here, the experimental outcomes show efficiency via $2.7 \%$ compared to the traditional process of LBPs.

Belhouchette et al. [35] have suggested a novel strategy to recognize fundamental emotions on image progression. The emotion of interest and generated their equivalent Action Units (AU) depending on psychology basics. Finally, the suggested method classified the outcomes with Kohonen Self Organizing Map (SOM)

Wei [36] have presented a new facial recognition algorithm on uncontrolled environment that combines Local Binary Pattern of Central Block Symmetry (CS-LBP) and the Deep Residual Network (DRN) form. The experimental results displayed the recognition rate and its superiority.

Zhang [37] have presented a dynamic face recognition approach based on block sample feature. According to the principal component feature decomposition approach, each block feature quantity was planned into foundation coordinates of test face sample set. The simulation outcomes portray that the algorithm had better feature matching and high recognition accuracy.

Putri et al. [38] have introduced a face recognition algorithm depending on block sample feature matching. According to principal component feature decomposition algorithm, each block feature quantity was planned into base coordinates of test face sample set. The simulation outcomes portray that the introduced algorithm had better feature matching and high recognition accuracy.
Li [39] have presented a gray relational analysis system. Here, the face image was considered a major investigation topic, an image recognition model depends on the gray relational analysis system was established and linked experimental outcomes were arriving. Compared with the traditional facial image recognition system, the presented system had greater recognition speed and good recognition efficiency.

Baker et al. [40] have presented an effectual system of facial recognition. Here, features were removed with PCA to diminish the dimensionality of facial images. FFBBL provides accuracy of $(98.33,98.80)$ with $(40,50)$ characteristics, while Elman provides $(98.33,95.14)$ with $(40,50)$ characteristics.

Shi and Tang [41] have presented a facial recognition approach depending on self-adaptable LBP, dual channel Convolutional Neural Network (CNN) using diverse convolutional nuclei. The outcomes portrayed that the presented system had a great respect rate as well as calculation performance.

$\mathrm{Li}$ and Cui [42] have presented 3D image reconstruction system of human maxillofacial defects based on lump model features matched. A human maxillofacial image features a matched pattern was built; 3-dimensional edge contour segmentation were executed for model images using blocking system. The simulation outcomes proved that the presented system contains better capability to detect and detect features and can provide high accuracy.

Nhat and Hoang [43] have presented a face recognition research topic on machine vision due to its highly secure demands. Here, the feature fusion was used with canonical association analysis to concatenate two dissimilar feature sources for encoding the facial image. Three descriptors (Histograms of Oriented Gradients (HOG), LBP, Global Descriptors (GIST)) were researched to extract the facial features based on block division.

Tran-Trung and Hoang [44] have presented hand gesture recognition applications at current years, like robotics, electronic commerce, human-machine interaction, electronic sports, and assistance to people with hearing disabilities. The document presents a strategy to hand gesture recognition in multi-view cameras. The presented strategy was assessed at HGM-4 reference dataset with LBPs.

Qin et al. [45] have presented a novel opinion on a few cases of different representations of facial images were helpful with facial recognition and 
appropriately diminishing that image resolution could be helpful for better classification of facial images. Furthermore, the idea appears to be helpful in serving people enhance facial recognition systems at real world.

Mi et al. [46] have introduced PCA depend strong feature extraction methods to treat the image with its transferred vector, leading to the latent information loss performed with images. PCA depending on block norm minimal using block norm. Performance was assessed with multiple datasets and outcomes were likened to other PCA-based methods.

Liu [47] have presented a feature recognition system for related human face key opinions. The experimental outcomes show the recognition time of suggested system was $<0.7 \mathrm{~s}$, SNR $>24 \mathrm{~dB}$, the recognized accuracy was $>90 \%$.

Mehta et al. [48] have presented a development of automated assistance systems based on facial recognition. The relative study was performed to choose suitable detection, classified approaches as faster Region-based Convolutional Neural Network (R-CNN) as well as SVM classification outperformed its equivalent competitors.

Aiordachioaie et al. [49] have established thermal imaging of human faces of categorization purposes.
Several data transforms were deemed with image processing, accompanied via information-based transformations, classification was considered. The result displayed that flexibility of toolbox depends on Discrete Cosine Transform (DCT) for feature selection.

Gnouma et al. [50] have suggested a supervised form via unsupervised learning with auto-encoder principle. Novel foreground detection depending on information removed as Gaussian Mixture Model (GMM) integrates uniform movement of optical flow magnitude. The outcomes demonstrate that the performance of the suggested strategy regarding with an irregularity in the performance of a stock, distortion of the form, point change, scale important changes.

Majhi and Pal [51] have presented retrieval scheme of image using block-level hybrid feature. The 1st level characteristics were created the DCT application; the 2nd-level characteristics were arrived that Singular Value Decomposition (SVD) processed. For the recovery mechanism, the similarity was scaled with five existing distance measurement systems to verify the performance. Table 1 shows the Comparative analysis of literature review.

Table 1 Comparative analysis of literature review

\begin{tabular}{|c|c|c|c|c|}
\hline Author and year & Method & Advantages & Disadvantages & Performance \\
\hline Huang et al. [34] & $\begin{array}{l}\text { Human emotion } \\
\text { recognition depends } \\
\text { on facial expression } \\
\text { detection with the } \\
\text { deep belief network }\end{array}$ & $\begin{array}{l}\text { Accurately recognize } \\
\text { the face }\end{array}$ & $\begin{array}{l}\text { Human face images } \\
\text { greatly in real } \\
\text { environments based on } \\
\text { difficult backgrounds and } \\
\text { luminance }\end{array}$ & $\begin{array}{l}\text { Face detection, FER } \\
\text { was accomplished } \\
\text { successfully }\end{array}$ \\
\hline $\begin{array}{l}\text { Belhouchette et al. } \\
{[35]}\end{array}$ & $\begin{array}{l}\text { Interest emotion } \\
\text { recognition strategy } \\
\text { with self-organising } \\
\text { map along with } \\
\text { motion estimation }\end{array}$ & $\begin{array}{ll}\text { Optimize } & \text { the } \\
\text { calculation time } & \text { and } \\
\text { enhance } & \text { that } \\
\text { recognition rate } & \end{array}$ & $\begin{array}{l}\text { Number of resources and } \\
\text { calculation time are } \\
\text { increased }\end{array}$ & $\begin{array}{l}\text { Accurately recognize } \\
\text { the face }\end{array}$ \\
\hline Wei [36] & $\begin{array}{l}\text { An innovative face } \\
\text { recognition of non- } \\
\text { controlled } \\
\text { environment }\end{array}$ & $\begin{array}{l}\text { Detecting the identity } \\
\text { of individuals to } \\
\text { monitor systems, } \\
\text { security }\end{array}$ & Poor accuracy & $\begin{array}{l}\text { The face detection, } \\
\text { FER } \\
\text { accomplished } \\
\text { positively }\end{array}$ \\
\hline Zhang [37] & $\begin{array}{l}\text { Improvement of face } \\
\text { recognition } \\
\text { technology based on } \\
\text { intelligent image }\end{array}$ & $\begin{array}{l}\text { Robust to the change } \\
\text { of skin color and pose }\end{array}$ & $\begin{array}{l}\text { The corner detection and } \\
\text { texture matching of the } \\
\text { face are carried out }\end{array}$ & $\begin{array}{l}\text { The simulation } \\
\text { outcomes show that } \\
\text { the system had better } \\
\text { feature matching and } \\
\text { high recognition }\end{array}$ \\
\hline Putri [38] & $\begin{array}{l}\text { Indonesian ethnicity } \\
\text { recognition depends } \\
\text { on face image with } \\
\text { Uniform Local Binary } \\
\text { Pattern (ULBP) }\end{array}$ & $\begin{array}{l}\text { Good feature } \\
\text { matching and high } \\
\text { recognition accuracy }\end{array}$ & High calculation time & $\begin{array}{ll}\text { Face detection and } \\
\text { FER } & \text { was } \\
\text { accomplished } & \\
\text { successfully } & \end{array}$ \\
\hline
\end{tabular}


International Journal of Advanced Technology and Engineering Exploration, Vol 8(83)

\begin{tabular}{|c|c|c|c|c|}
\hline Author and year & Method & Advantages & Disadvantages & Performance \\
\hline $\operatorname{Li}[39]$ & $\begin{array}{l}\text { Image Recognition } \\
\text { depends on grey } \\
\text { relational analysis }\end{array}$ & $\begin{array}{ll}\text { High } & \text { recognition } \\
\text { accuracy } & \end{array}$ & $\begin{array}{l}\text { Poor classification } \\
\text { accuracy }\end{array}$ & $\begin{array}{l}\text { Higher recognition } \\
\text { speed and good } \\
\text { recognition } \\
\text { performance }\end{array}$ \\
\hline Baker et al. [40] & $\begin{array}{l}\text { Facial recognition } \\
\text { enhancement using an } \\
\text { artificial neural } \\
\text { network by PCA }\end{array}$ & $\begin{array}{l}\text { Detect } r \text { people's } \\
\text { identity to monitor } \\
\text { systems, security and } \\
\text { numerous practical } \\
\text { fields }\end{array}$ & $\begin{array}{l}\text { Diminish the } \\
\text { dimensionality of face } \\
\text { image }\end{array}$ & $\begin{array}{l}\text { The outcomes show } \\
\text { that the presented } \\
\text { system was effective } \\
\text { and extremely } \\
\text { accurate }\end{array}$ \\
\hline Shi and Tang [41] & $\begin{array}{lr}\text { Face recognition } \\
\text { approaches depends } \\
\text { on self-adaptive } \\
\text { blocking LBP }\end{array}$ & $\begin{array}{l}\text { To recover } \\
\text { successfully the face } \\
\text { recognition rate }\end{array}$ & $\begin{array}{l}\text { The distribution of the } \\
\text { bright, dark spot and } \\
\text { other micro details } \\
\text { cannot be completely } \\
\text { fulfilled }\end{array}$ & $\begin{array}{l}\text { The outcomes show } \\
\text { that the presented } \\
\text { system had great } \\
\text { recognition rate and } \\
\text { computational } \\
\text { efficiency }\end{array}$ \\
\hline $\mathrm{Li}$ and Cui [42] & \begin{tabular}{lr}
$3 \mathrm{D}$ & image \\
reconstruction & system \\
of & human \\
maxillofacial defect & imaging depends on \\
matching block & \multicolumn{2}{l}{ sample features } \\
\end{tabular} & $\begin{array}{l}\text { Three-dimensional } \\
\text { image recognition and } \\
\text { reconstruction of } \\
\text { human maxillofacial } \\
\text { defects }\end{array}$ & $\begin{array}{lr}\text { Human face } & \text { image } \\
\text { sample } & \text { template } \\
\text { matching, edge } & \text { contour } \\
\text { segmentation } & \text { is } \\
\text { mistreated } & \end{array}$ & $\begin{array}{l}\text { The simulation } \\
\text { outcomes show that } \\
\text { the presented system } \\
\text { contains, the better } \\
\text { ability to detect and } \\
\text { detect features and can } \\
\text { provide high precision }\end{array}$ \\
\hline Nhat and Hoang [43] & $\begin{array}{l}\text { Function fusion with } \\
\text { HOG, LBP, GIST } \\
\text { descriptors along with } \\
\text { canonical correlation } \\
\text { analysis of facial } \\
\text { recognition }\end{array}$ & $\begin{array}{l}\text { Improve the accuracy } \\
\text { of face recognition } \\
\text { systems }\end{array}$ & $\begin{array}{ll}\text { Poor } & \text { classification } \\
\text { accuracy } & \end{array}$ & $\begin{array}{l}\text { to recover efficiently } \\
\text { that face recognition }\end{array}$ \\
\hline $\begin{array}{l}\text { Tran-Trung } \\
\text { Hoang [44] }\end{array}$ & $\begin{array}{lr}\text { Hand } & \text { gesture } \\
\text { recognition } & \text { in } \\
\text { Multiview } & \text { cameras } \\
\text { with local } & \text { image } \\
\text { descriptors } & \\
\end{array}$ & $\begin{array}{l}\text { Good feature } \\
\text { matching and high } \\
\text { recognition accuracy }\end{array}$ & High calculation time & $\begin{array}{l}\text { Face detection and } \\
\text { FER } \\
\text { accomplished were } \\
\text { successfully }\end{array}$ \\
\hline Qin et al. [45] & $\begin{array}{lr}\text { Discover alternative } \\
\text { representations of } \\
\text { facial images with } \\
\text { facial recognition }\end{array}$ & $\begin{array}{l}\text { Browse high } \\
\text { resolution face images } \\
\text { for better image } \\
\text { ranking }\end{array}$ & $\begin{array}{l}\text { The amount of used } \\
\text { resources and calculation } \\
\text { time is increased }\end{array}$ & $\begin{array}{l}\text { Useful to help people } \\
\text { improve facial } \\
\text { recognition }\end{array}$ \\
\hline Mi et al. [46] & $\begin{array}{l}\text { Block-norm } \\
\text { minimization based } \\
\text { PCA }\end{array}$ & $\begin{array}{l}\text { Good feature } \\
\text { matching and high } \\
\text { recognition accuracy }\end{array}$ & $\begin{array}{l}\text { Latent information loss } \\
\text { performed with images }\end{array}$ & $\begin{array}{l}\text { It was efficiently } \\
\text { depress the effect of } \\
\text { corrupt blocks. }\end{array}$ \\
\hline Liu [47] & $\begin{array}{l}\text { A feature recognition } \\
\text { system for related } \\
\text { human face key points } \\
\text { depends on the } \\
\text { adaptive median filter }\end{array}$ & $\begin{array}{l}\text { To eliminate that the } \\
\text { image influence } \\
\text { background on related } \\
\text { key feature points } \\
\text { recognition }\end{array}$ & $\begin{array}{l}\text { Low efficiency and poor } \\
\text { accuracy }\end{array}$ & $\begin{array}{l}\text { Recognition accuracy } \\
\text { was more than } 90 \% \text {, } \\
\text { recognition good } \\
\text { results. }\end{array}$ \\
\hline Mehta et al. [48] & $\begin{array}{l}\text { Real time image } \\
\text { processing: integrated } \\
\text { face recognition based } \\
\text { automated attendance } \\
\text { system }\end{array}$ & $\begin{array}{ll}\text { High } & \text { recognition } \\
\text { accuracy } & \end{array}$ & $\begin{array}{ll}\text { Poor } & \text { classification } \\
\text { accuracy } & \end{array}$ & $\begin{array}{l}\text { Higher recognition } \\
\text { speed and good } \\
\text { recognition } \\
\text { performance }\end{array}$ \\
\hline $\begin{array}{l}\text { Aiordachioaie et al. } \\
\text { [49] }\end{array}$ & $\begin{array}{l}\text { On processing, } \\
\text { thermal images of } \\
\text { human faces for } \\
\text { classification purposes }\end{array}$ & $\begin{array}{l}\text { Detecting that identity } \\
\text { of individuals for } \\
\text { monitoring systems }\end{array}$ & $\begin{array}{l}\text { Correct representations } \\
\text { and descriptors / } \\
\text { characteristics of great } \\
\text { recognition }\end{array}$ & $\begin{array}{l}\text { The outcomes were } \\
\text { hopeful and exhibit } \\
\text { the flexibility }\end{array}$ \\
\hline Gnouma et al. [50] & $\begin{array}{lr}\text { Stacked } & \text { sparse } \\
\text { autoencoder } & \text { and } \\
\text { binary motion image }\end{array}$ & $\begin{array}{l}\text { Recover that accuracy } \\
\text { of facial recognition } \\
\text { systems }\end{array}$ & Minimum efficiency & $\begin{array}{l}\text { The outcomes } \\
\text { demonstrate that the } \\
\text { efficiency of presented } \\
\text { strategy regarding } \\
\text { within discretion }\end{array}$ \\
\hline
\end{tabular}


Pravin B Chopade and Prabhakar N

\begin{tabular}{lllll}
\hline Author and year & Method & Advantages & Disadvantages & Performance \\
\hline & & & & under the performance \\
\hline Majhi and Pal [51] & The image retrieval & Robust to the change & the corner detection and & The \\
& system depends on & simulation \\
& block level hybrid & & face are carried out tor and pose & outcomes show that \\
DCT-SVD fused & & & $\begin{array}{l}\text { algorithm had great } \\
\text { recognition accuracy }\end{array}$ \\
\hline
\end{tabular}

\subsection{Research gap}

One of a challenging research topic is human facial recognition method. The FER approaches contain GWT, AAM, and LBP. The GWT technique extracted face imagery texture information in several scales with direction attains better outcomes. But, the cost of time and the complexity of space is high. The facial expression dynamics can capture through analyzing locations of signs from AAM that is utilized for extracting shape feature. Though AAM can effectively get facial feature points, the fitting approach has a non-linear optimized issue, which has increased the calculation process to a more complex as well as computational complexity.

To overcome these issues, human emotion recognition based on block patterns of image and DWT is proposed. Here, DWT is utilized to extract the features of facial image for proposed algorithm due to its good localization characteristic. Before the application of DWT to an image whose emotions are to be identified, the image is divided into different block patterns, which is utilized in the remaining processing of the proposed algorithm. Among different evaluation parameters such as energy, covariance, correlation coefficients, etc., the dominant parameter energy is used to determine the emotions of the text image from the reference image. Finally, the proposed method increases the accuracy with less complexity during the face image recognition.

\section{System description and methodology}

DWT is proposed to capture the faces differences. Facial recognition can be done utilizing thresholding the range depending on the contact. Here, the wavelet change was motivated in the direction of light, facial pose and facial expression. The experiments utilized contact as well as start values to ensure greater consistency of product classification results. Encouraging test outcomes, the proposed algorithm is a viable and effective solution for recognizing faces, which leads to, well, practical use of forensic databases that exist in computerized human facial recognition applications. Figure 1 shows the Block diagram of the proposed method.
The proposed system for emotion recognition is classified into three parts.

1. Block pattern of the image (text/reference image)

2. Use of DWT

3. Extraction of features and its comparison

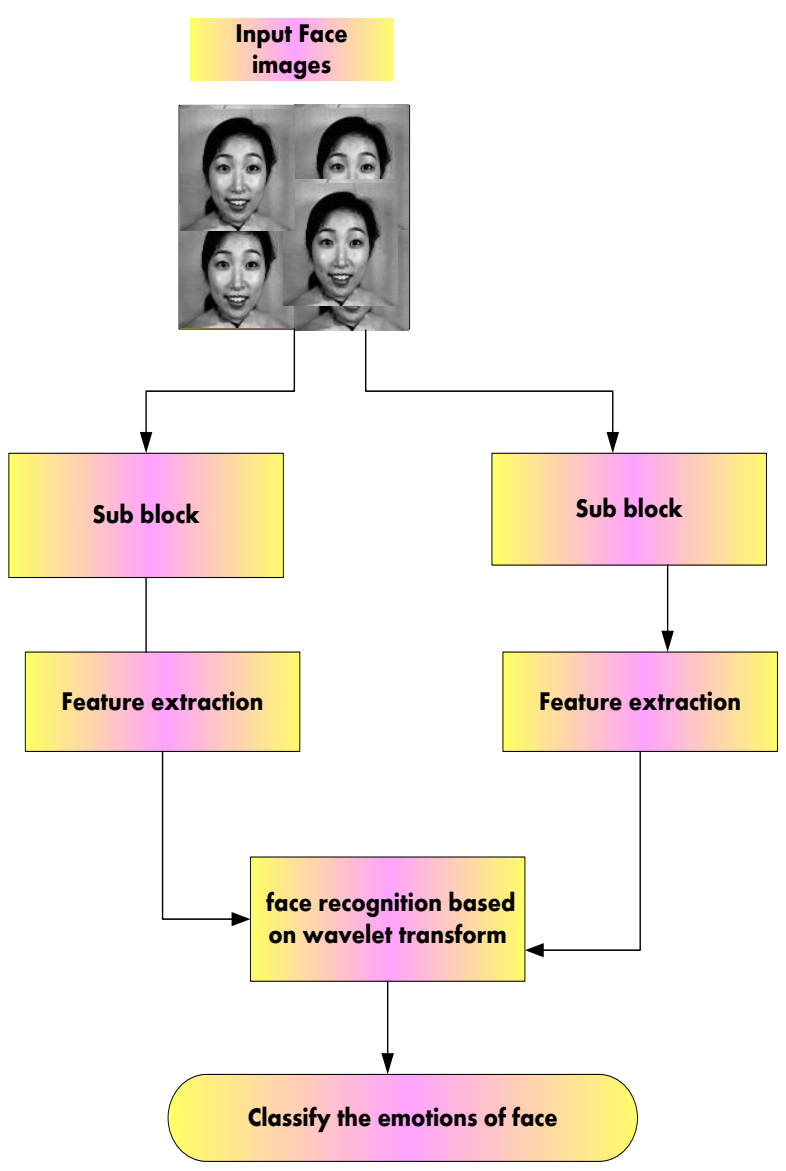

Figure 1 Block diagram of the proposed method

\subsection{Block pattern of the image (text /reference image)}

Normally, the face is a part of the body that is either used in face recognition techniques or emotion recognition systems. Face recognition is comparatively easy as in emotions micro expressions are involved that remain on for milliseconds or microseconds to face. 
Thus, capturing the micro expression is a little tedious and complicated. Furthermore, muscle movements of the face play a vital role in understanding emotions, and these muscle movements vary from person to person. However, the changes in emotion employ a change in the positions of muscles that generate different variations in the lines which are formed on the face. These formed lines change the energy of a specific part of the face. Hence, in the proposed algorithm, before feature extraction by DWT, the face (emotions to be recognized) is divided into specific block patterns which are utilized to measure different parameters such as energy, covariance, correlation coefficient etc.

The division of block pattern is elaborated as follows:

1. Text image is initially divided into two parts vertically and horizontally, named as $\mathrm{P}_{1}, \mathrm{P}_{2}$ and $\mathrm{P}_{3}$, $\mathrm{P}_{4}$, respectively.

2. Again, the blocks of images $P_{1}$ and $P_{2}$ are further divided vertically and horizontally into two parts, namely, $\mathrm{P}_{5}, \mathrm{P}_{6}$ and $\mathrm{P}_{7}, \mathrm{P}_{8}$, respectively.

3. In this fashion, each block of the image is divided vertically and horizontally into sub-blocks.

4. This division of the block may extend to a large number of sub-blocks, but in this proposed algorithm, it is limited up to 12 by extensive observation. The block division hierarchy is shown in Figure 2.

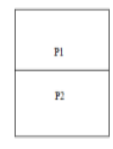

a. Division of Test image

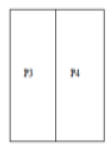

Figure 2 pattern

\subsection{Detailed description of DWT algorithm for face reorganization}

In this manuscript, the DWT is generally utilized Transform in image process. This DWT method provides high accuracy, high speed with less computational complexity and DWT is capturing the differences in faces. Face recognition depends on contact can be done through utilizing threshold.

The DWT decay an image as a set of depended functions named wavelet; decay is defined as "resolution" of imagery. DWT acts a signal multiple resolution analysis along localization in both times, frequency domain. The 2D-DWT filter is applied as a set of banks, which includes a continuous plan of 1401 high and pass filters. The final outcomes derived are a decay of input image as 4 non-overlapping multiresolution sub bands: LL, LH, HL, HH. The sub-band LL signifies coarse-scale DWT coefficients, subbands LH, HL, HH denote the fine-scale of DWT coefficients. HH is discovered for face recognition.

The detailed explanations of the DWT algorithm are given below:

Step1. Every face reorganization image decomposes with the help of DWT.

Step2. HH band of decayed imagery is utilized to more process.

Step3. HH band resizes to original image size.

Step4. Every resizing image is divided into sub images.

Step5. Convert every sub picture as a column data matrix. Each of them expresses in order to D-by-N. Then the decomposition equation is given as $K_{j}=\left\{k_{j 1}+k_{j 1}+\ldots k_{j M}\right\}, j=1,2, . . L, M \quad$ is represented as the total count of images.

Step6. Calculate the average value for each subimage.

Step7. Subtract the average value from the column data matrix of every subimage, then vertically centralized column data matrix as $K_{n j}=\left\{\vec{k}_{j 1}+\vec{k}_{j 1}+\ldots \vec{k}_{j M}\right\}, j=1,2, . . L$

Step8. Rearrange components to obtain square matrix. Step9. Gather Eigen values, Eigenvectors, diagonal values of square matrix as, $H_{j}=\left\{H_{j 1}+H_{j 1}+\ldots H_{j P}\right\}, j=1,2, . . L$,

Here, $P$ is represented as the sub image feature. Then attain trained data base matrix $Y_{m j}=L_{x j}{ }^{T} Z_{x j}=\left\{Y_{j 1}+Y_{j 2}+\ldots Y_{j P}\right\}=1,2, . L$

Step10. Replication same process for row data matrix. Step11. Lessen the required feature size $Y_{m j}=\left\{Y_{i 1}{ }^{T}+Y_{i 2}{ }^{T}+\ldots Y_{i P}{ }^{T}\right\}, i=1,2, . L$

Step12. The steps above are repeated to create feature 2 for the entire picture without DWT.

Step13. Minkowski distance is utilized recover related images

Step14. Minkowski distance focuses on Euclidean space that is measured as both Euclidean generalization, Manhattan distance for receiving efficiency of more recognition. The Minkowski distance depends upon factor $L$.

$L=\left(a_{1}, a_{2}, \ldots . a_{m}\right)$

Minkowski distance is utilized through $\mathrm{p}$ existence 1 or 2. In limited case of $L$ attainment infinity gets the Chebyshev distance. 


$$
F_{d}(x, z)=P_{L}(x, z)=\left(\sum_{a=1}^{m}\left|a_{j}-b_{j}\right|^{l}\right)^{j / l}
$$

Minkowski distance is frequently utilized when variables have been scaled on ration scales bycomplete0value.

\subsection{Extraction of features and comparisons}

The different evaluator parameter which is derived from the frequency sub band of each block of text and reference image after application of wavelet transform is compared.

This evaluation is carried out in unique and specific ways, so the maximum match is obtained from the text and reference image based on different parameters. Based on this comparison, a conclusion is determined about the classification of emotions, like happiness, anger, sadness, etc. The comparative blocks of different patterns are shown in Tables 2, 3, and 4,5 .

Table 2 Basic block comparison

\begin{tabular}{ll}
\hline Test imagery blocks & Reference imagery blocks \\
\hline P1 & P1 \\
\hline & \\
\hline P2 & P2 \\
\hline P3 & P3 \\
\hline P4 & P4 \\
\hline
\end{tabular}

Table 3 Block comparison of P1/P2 test imagery

\begin{tabular}{ll}
\hline $\begin{array}{l}\text { Test imagery blocks of } \\
\text { P1/P2 }\end{array}$ & Reference imagery blocks \\
\hline P5 & P1/P2 \\
\hline P6 & P1/P2 \\
\hline P7 & P1/P2 \\
\hline P8 & P1/P2 \\
\hline
\end{tabular}

Table 4 Block comparison of P3/P4 test image

\begin{tabular}{ll}
\hline Test imagery blocks & Reference imagery blocks \\
\hline P9 & P3/P4 \\
\hline P10 & P3/P4 \\
\hline P11 & P3/P4 \\
\hline P12 & P3/P4 \\
\hline
\end{tabular}

3.4Face recognition depends on wavelet transform Algorithm

Here, the document, depending on wave transformation and face recognition of PCA block, indicated with Wavelet Transform on Block PCA (WT-BPCA) that has 2 processes: trained and recognized process. The exact method as below:

A. Training process
(1) Initially, the images of faces are carried out wavelet transform.

(2) To process that image subspace based on the traditional PCA, the entire column is linked.

$\left.C j=A_{j} A_{j} T, \quad j=1,2,3 \ldots r\right)$

Here, $A_{j}=[\rightarrow \Phi 1 \mathrm{j}, \rightarrow \Phi 2 \mathrm{j}, \ldots, \rightarrow \Phi \mathrm{Lj}]$,

(3) Compute $\mathrm{Cj}$ to obtain eigenvector $\mathrm{Uj}=[\mathrm{u} \rightarrow \mathrm{j} 1, \mathrm{u}$ $\rightarrow \mathrm{j} 2, \ldots, \mathrm{u} \rightarrow \mathrm{jm}]$ with SVD.

(4)To obtain that projection coefficient $\mathrm{mj}$,

(5)Receive combined projection coefficient $\mathrm{m}$, here $\mathrm{m}$ is: $m=1 \mathrm{r} \mathrm{j}=\sum w j m j$

B. Recognition process

(1) In the trained process, the face imageries denote wave transforms, after removing minimum-frequency coefficients.

(2) Projected very block with equivalent eigenvectors and projection coefficient $\Psi \mathrm{j}(\mathrm{j}=1,2, \ldots, \mathrm{r})$.

(3) Obtain incorporated projection coefficient $\Psi, \Psi=$ $1 \mathrm{r} \mathrm{j}=\sum \mathrm{w} \mathrm{j} \Psi \mathrm{j}$ (11) here $\Psi \mathrm{j}$ specifies sub-space image weight coefficient.

\subsection{Databases}

In this work, the proposed model takes 4 FER datasets, such as FER 2013 [52], the extended CohnKanade (CK+) [53], Japanese Female Facial Expression (JAFFE) [54], Facial Expression Research Group Database (FERG) [55].

FER2013: This is first developed in ICML 2013 challenges in representation learning [14]. It contains 32, 298 imageries of $48 \times 48$ resolution, mostly taken from wild settings. Initially, the training set contains 28,709 imageries, then the validation with test sets involves 3589 imageries. This dataset is generated by Google image search Application Programming Interface (API), then the faces are registered automatically. The faces are labelled as any 6 cardinal expressions along neutral. When likened with other datasets, FER has lots of variation in the imageries, via facial occlusion (mostly with a hand), partial faces, low-contrast images, eyeglasses.

$\mathbf{C K}+$ : This is a public dataset for the action unit together with emotion recognition. It contains posed and non-posed (spontaneous) expressions. A total of 593 sequences 400 images for training and 193 for testing.

JAFFE: It consists of 213 imageries of 7 facial expressions posed by 10 Japanese female models. In this dataset, 213 images were used, 180 images for 
training and 33 for testing. Every image is rated in 6 emotional adjectives by 60 Japanese themes [15].

FERG: This is a stylized character database with annotated facial expressions. It has 55,767 annotated face imageries of 6 stylized characters. The characters are modeled through MAYA. Originally, the training set has 50,767 imageries, and then the validation with test sets has 5000 imageries.

\section{Results}

Here, the simulation performance the HER-BP-DWT is proposed. The proposed method is simulated using MATLAB. Here, evaluation metrics, viz accuracy, sensitivity, specificity and precision are analysed. The performance of the proposed approach is likened with two existing approaches, like HER-CNN [56], and HER-BFA [57].
Figure 3 depicts the test image. Here, the different block patterns named P1, P2 and P3, P4 horizontally and vertically respectively. The DWT applies to these block patterns that result from various frequency subbands: LL, LH, HL, HH. The coefficients of subbands are either integer numbers or floating numbers. The energies of these sub-bands are measured and utilized for the determination of the particular emotion based on the match with the energy of the blocks of the reference image. The Energy components of different test and reference images and frequency sub bands of blocks P1, P2 and P3, P4 are shown in Table 5. The test image provides smiley expressions. These expressions are substantially reflected in the blocks $\mathrm{P} 2$ and $\mathrm{P} 4$ so that it gives more information regarding smiley expression. Due to more smiley expressions in blocks $\mathrm{P} 2$ and P4, so the energy components of the same area.

Table 5 Energy components of different test and reference images

\begin{tabular}{llllll}
\hline Block & Pic1 & Pic2 & Pic 3 & Pic 4 & Pic 5 \\
\hline P1 & 4.6275 & 4.4232 & 4.2828 & 3.3676 & 4.0607 \\
\hline P2 & 4.4274 & 4.6045 & 4.5259 & 3.4538 & 4.7435 \\
\hline P3 & 4.0210 & 4.1100 & 4.0137 & 2.69255 & 4.0348 \\
\hline P4 & 5.0338 & 4.9176 & 4.7950 & 4.1959 & 4.7685 \\
\hline P5 & 4.5478 & 3.7611 & 3.6875 & 2.4487 & 3.9711 \\
\hline P6 & 4.7043 & 5.4478 & 5.3643 & 4.5988 & 5.5140 \\
\hline P7 & 5.3857 & 5.2213 & 5.1945 & 3.8919 & 5.1955 \\
\hline P8 & 3.8693 & 3.9876 & 3.8573 & 3.1556 & 4.2895 \\
\hline P9 & 4.7073 & 4.3875 & 4.2258 & 3.7930 & 4.0240 \\
\hline P10 & 5.3604 & 5.4478 & 5.3643 & 4.5988 & 5.5140 \\
\hline P11 & 5.4223 & 5.2719 & 5.2416 & 4.4025 & 4.9484 \\
\hline P12 & 4.6454 & 4.5634 & 4.3485 & 3.9893 & 4.5883 \\
\hline
\end{tabular}

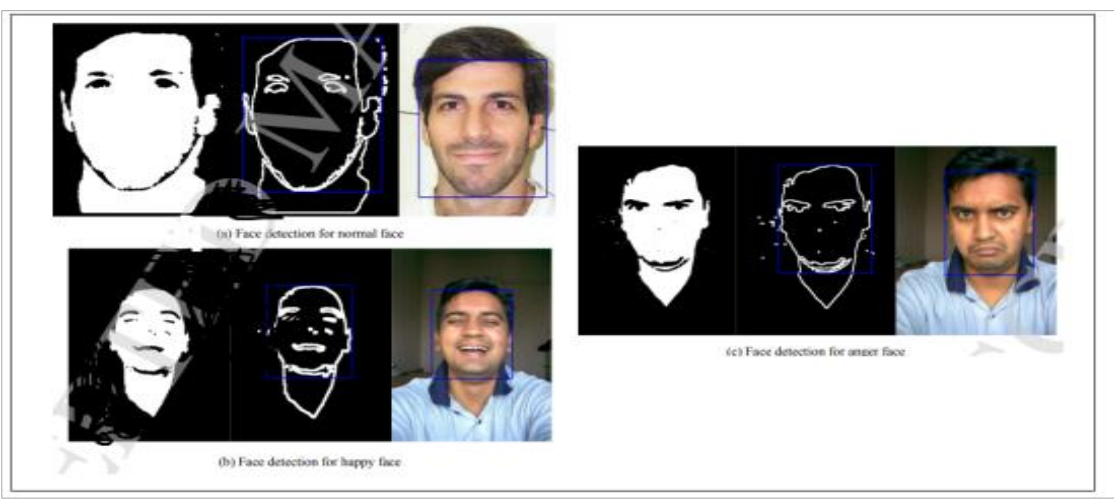

Figure 3 Face recognition using DWT

Similarly, the reference image is subdivided into different blocks and its decomposition is carried out utilizing DWT that results in various frequency sub bands.
The experimentations are carried out using different test images which show different emotions, such as happiness, anger, sadness, etc. named Pic1to Pic5. The test images are used for experimentation are shown in Figure 4. Here, the energy details of the 
decomposed components are collected in the database, and these energy components are employed for recognizing that emotions by matching energy components of the test and reference image. The segmentation of reference image and the decomposition of its blocks into different frequency sub bands. Table 6 shows, training and testing of the face recognition using DWT. On observing the table, the Accuracy, Precision, Sensitivity, Specificity of proposed BD-DWTA method shows better than the existing methods, such as human face recognition using HER-CNN and human face recognition using HER-BFA. Here, accuracy shows $99.55 \%$, sensitivity shows $85.93 \%$, Precision shows $92.43 \%$, Specificity shows $90.74 \%$, higher than the existing methods, such as human face recognition using HER-CNN and human face recognition using HER-BFA.

Table 6 Comparison training and testing results face recognition using DWT

\begin{tabular}{lllll}
\hline Methods & Accuracy & Precision & Sensitivity & Specificity \\
\hline HER-CNN & $83.55 \%$ & $75.49 \%$ & $70.84 \%$ & $80.54 \%$ \\
\hline HER-BFA & $87.64 \%$ & $77.85 \%$ & $75.63 \%$ & $73.52 \%$ \\
\hline $\begin{array}{l}\text { HER-BP-DWT } \\
\text { (Proposed) }\end{array}$ & $99.55 \%$ & $92.43 \%$ & $85.53 \%$ & $90.74 \%$ \\
\hline
\end{tabular}

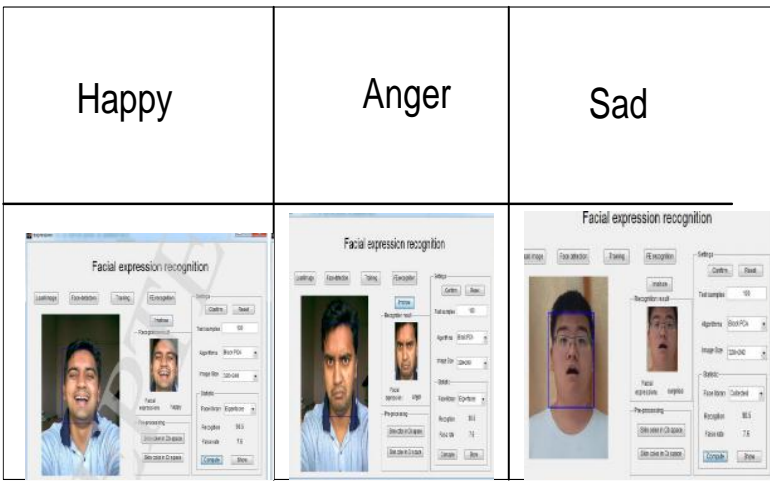

Figure 4 Face recognition of happy, sad and anger using DWT

\subsection{Software and hardware implementation of face reorganization}

Figure 5 shows the Software and hardware Implementation of the face reorganization system. To make the automatic door access system, the personal computer (PC) RS232 converter is linked through microcontroller via USB. When no face is detected in front of webcam, no signal is sent to the microcontroller. The door is closed because microcontroller does not receive any signal from PC, when a face is detected the name of the authorized person is displayed in the left corner of the noticing box in MATLAB GUI. Once the face is recognized, the door is opened automatically.

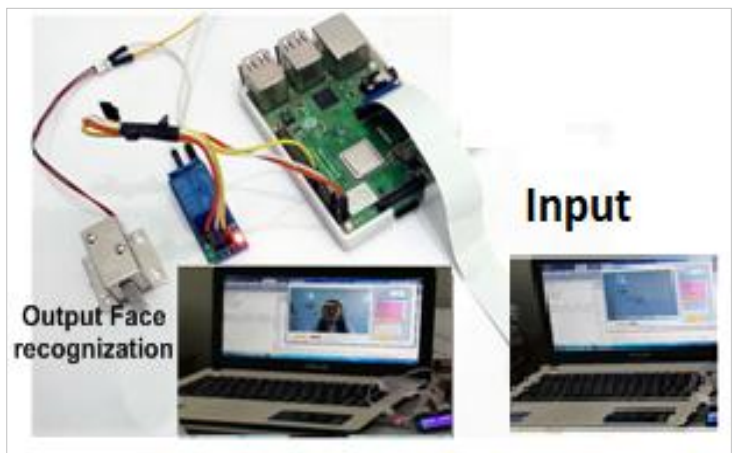

Figure 5 Software and hardware implementation of face reorganization

Figure 6 shows the accuracy, sensitivity, specificity and precision of FER2013 dataset using human face recognition-based pattern block and DWT method. Here, the HER-BP-DWT method is analyzed with two existing methods, such as HER-CNN, HERBFA. From the analysis, the proposed HER-BPDWT method has an accuracy of $82 \%$ and $89 \%$ higher than the existing methods, such as human face recognition using HER-CNN and human face recognition using HER-BFA. The proposed HERBP-DWT method has a precision of $80 \%$ and $89 \%$ higher than the existing method such as human face recognition using HER-CNN and human face recognition using HER-BFA. The proposed HERBP-DWT method has a sensitivity of $83 \%$ and $84 \%$ higher than the existing method such as human face recognition using HER-CNN and human face recognition using HER-BFA. 
The proposed HER-BP-DWT method has Specificity of $82 \%$ and $89 \%$ higher than the existing method such as human face recognition using HER-CNN and human face recognition using HER-BFA.

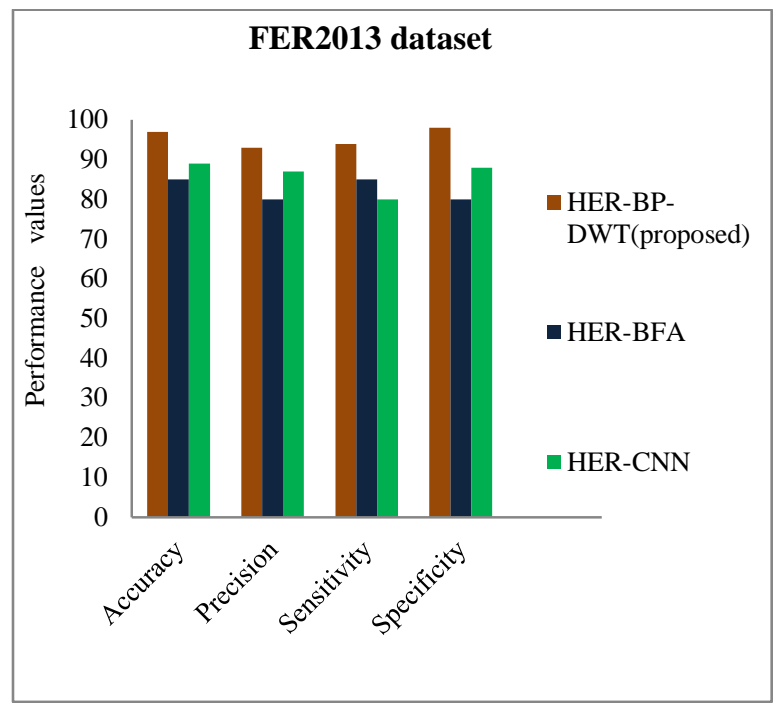

Figure 6 Performance metrics of FER2013 dataset

Figure 7 shows the accuracy, sensitivity, specificity and precision of $\mathrm{CK}+$ dataset using human face recognition-based pattern block and DWT method. Here, the HER-BP-DWT method is examined with two existing methods, such as HER-CNN, HERBFA. Here, the proposed HER-BP-DWT method has an accuracy of $80 \%$ and $89 \%$ higher than the existing method such as human face recognition using HER$\mathrm{CNN}$ and human face recognition using HER-BFA.

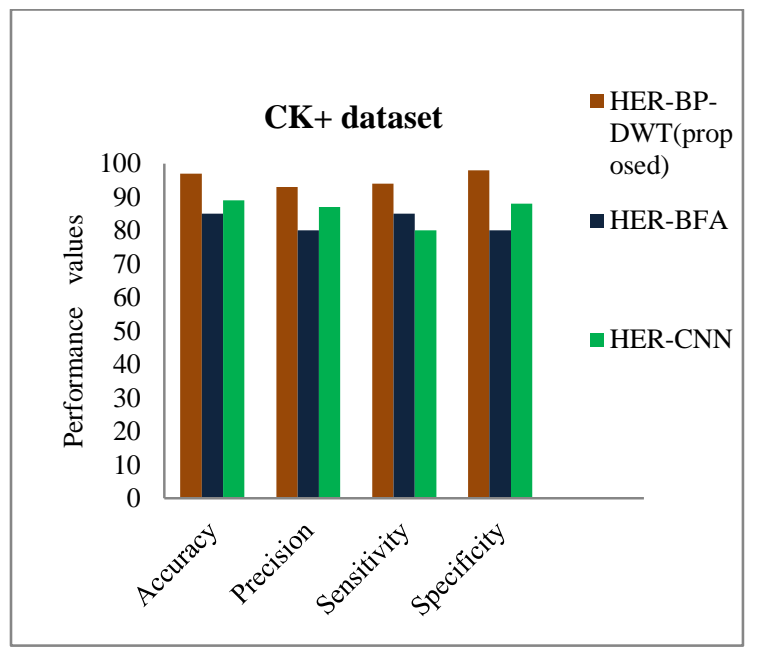

Figure 7 Performance metrics of $\mathrm{CK}+$ dataset

The proposed HER-BP-DWT method has a precision of $83 \%$ and $85 \%$ higher than the existing method 1405 such as human face recognition using HER-CNN and human face recognition using HER-BFA. The proposed HER-BP-DWT method has a sensitivity of $85 \%$ and $86 \%$ higher than the existing method such as human face recognition using HER-CNN and human face recognition using HER-BFA. The proposed HER-BP-DWT method has Specificity of $88 \%$ and $84 \%$ higher than the existing method such as human face recognition using HER-CNN and human face recognition using HER-BFA.

Figure 8 shows the accuracy, sensitivity, specificity and precision of JAFFE dataset using human face recognition-based pattern block and DWT method. Here, the HER-BP-DWT method is examined with two existing methods, such as HER-CNN, HERBFA. Here, the proposed HER-BP-DWT method attains accuracy of $82 \%$ and $84 \%$ higher than the existing methods, such as human face recognition using HER-CNN and human face recognition using HER-BFA.

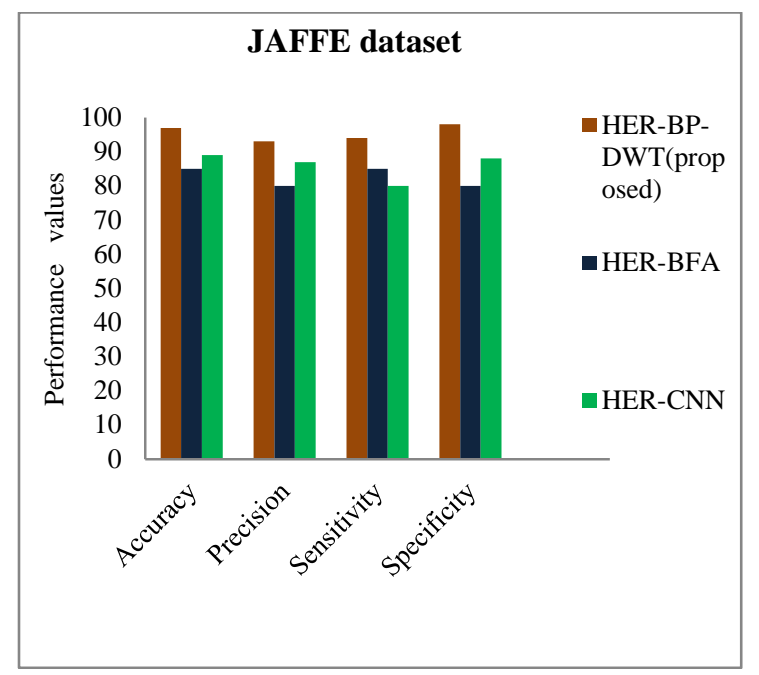

Figure 8 Performance metrics of JAFFE dataset

The proposed HER-BP-DWT method attains precision of $86 \%$ and $89 \%$ higher than the existing methods, such as human face recognition using HERCNN and human face recognition using HER-BFA. The proposed HER-BP-DWT method attains sensitivity of $84 \%$ and $82 \%$ higher than the existing methods, such as human face recognition using HERCNN and human face recognition using HER-BFA. The proposed HER-BP-DWT method attains Specificity of $87 \%$ and $86 \%$ higher than the existing methods, such as human face recognition using HER$\mathrm{CNN}$ and human face recognition using HER-BFA. 
Figure 9 shows the accuracy, sensitivity, specificity and precision of FERG dataset using human face recognition-based pattern block and DWT method. Here, the HER-BP-DWT method is analysed with two existing methods, such as HER-CNN, HERBFA. Here, the proposed HER-BP-DWT method attains $84 \%$ and $87 \%$ accuracy higher than the existing methods, such as human face recognition using HER-CNN and human face recognition using HER-BFA. The proposed HER-BP-DWT method attains $83 \%$ and $85 \%$ precision higher than the existing methods, such as human face recognition using HER-CNN and human face recognition using HER-BFA. The proposed HER-BP-DWT method attains $84 \%$ and $87 \%$ sensitivity higher than the existing methods, such as human face recognition using HER-CNN and human face recognition using HER-BFA. The proposed HER-BP-DWT method attains Specificity of $82 \%$ and $87 \%$ higher than the existing methods, such as human face recognition using HER-CNN and human face recognition using HER-BFA.

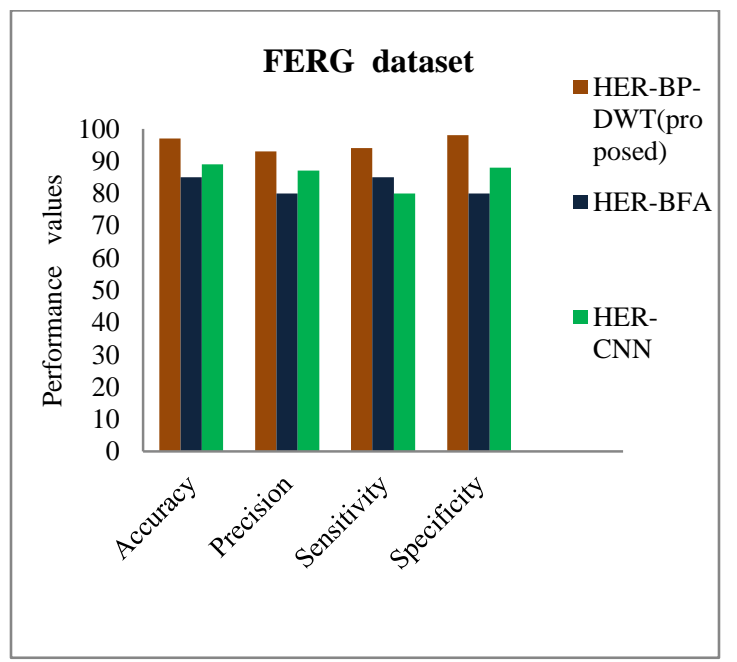

Figure 9 Performance metrics of FERG dataset

\section{Discussion}

In this method four types of datasets are used, that are FER 2013, the extended Cohn-Kanade, JAFFE, and FERG. These datasets are compared with the existing methods, HER-CNN, HER-BFA approach in terms of accuracy, sensitivity, specificity, precision. In FER2013 dataset, the accuracy of FER 2013 dataset is $82 \%$ and $89 \%$ higher than the existing methods. The precision of FER 2013 dataset is 80\% and 89\% higher than the existing methods. The sensitivity of FER2013 dataset is $83 \%$ and $84 \%$ greater than the existing models, Specificity of FER2013 dataset is $82 \%$ and $89 \%$ greater than the existing models, viz 1406 human face recognition using HER-CNN and human face recognition using HER-BFA.

The accuracy of CK+ dataset is $80 \%$ and $89 \%$ higher than the existing methods. The precision of CK+ dataset is $83 \%$ and $85 \%$ greater than the existing models. The sensitivity of $\mathrm{CK}+$ dataset is $85 \%$ and $86 \%$ greater than the existing models, Specificity of $\mathrm{CK}+$ dataset $84 \%$ and $86 \%$ greater than the existing models, such as human face recognition using HER$\mathrm{CNN}$ and human face recognition using HER-BFA.

In JAFFE dataset, the accuracy of JAFFE dataset is $82 \%$ and $84 \%$ higher than the existing methods. The precision of JAFFE is $86 \%$ and $89 \%$ higher than the existing methods. The sensitivity of JAFFE dataset is $84 \%$ and $82 \%$ greater than the existing models, Specificity of JAFFE dataset is $87 \%$ and $86 \%$ greater than the existing models, such as human face recognition using HER-CNN and human face recognition using HER-BFA.

In FERG dataset, the accuracy of FERG dataset is $84 \%$ and $87 \%$ higher than the existing methods. The precision of FERG is $83 \%$ and $85 \%$ greater than the existing models. The sensitivity of FERG dataset is $84 \%$ and $87 \%$ greater than the existing models, Specificity of FERG dataset is $82 \%$ and $87 \%$ greater than the existing models, viz human face recognition using HER-CNN and human face recognition using HER-BFA.

\section{Highlight of result}

- the performance metrics of accuracy for face recognition using HER-BP-DWT approach shows high accuracy $96.5 \%$

- the performance metrics of specificity for face recognition using HER-BP-DWT approach shows high specificity $94.5 \%$

- the performance metrics of sensitivity for face recognition using HER-BP-DWT approach shows high sensitivity $93.5 \%$

- the performance metrics of precision for face recognition using HER-BP-DWT approach shows high precision $92.5 \%$

- the proposed system is likened with two existing methods, viz HER-CNN and HER-BFA

\subsection{Limitations of this work}

With the face database raise, face recognized rate will suffer. The face databases contain more public databases. Thus, the significant direction of future research is to uphold recognition rate stability under high databases. 
A complete list of abbreviations is shown in Appendix I.

\section{Conclusion}

In this manuscript, an innovative method of block pattern is introduced for the recognition of different emotions. The block pattern is unique and simple that is followed in the proposed algorithm. The DWT is investigated to facial expression recognition. The energy components of different separated blocks are very much useful for the determination of the blocks match pattern, and hence different emotions are easily classified with proper accuracy and efficient way. The test image is classified into different kinds of emotions, such as sadness, disgust, cry, etc. efficiently. The experimental results show that the proposed BD-DWTA method is better than the existing methods. Here, accuracy shows 99.55\%, sensitivity shows $85.93 \%$, Precision shows $92.43 \%$, Specificity shows $90.74 \%$, higher than the existing methods, like HER-CNN [56] and HER-BFA [57].

\section{Acknowledgment}

None.

\section{Conflicts of interest}

The authors have no conflicts of interest to declare.

\section{References}

[1] Matsumoto D, Hwang HS. Evidence for training the ability to read microexpressions of emotion. Motivation and Emotion. 2011; 35(2):181-91.

[2] Quintana DS, Guastella AJ, Outhred T, Hickie IB, Kemp AH. Heart rate variability is associated with emotion recognition: direct evidence for a relationship between the autonomic nervous system and social cognition. International Journal of Psychophysiology. 2012; 86(2):168-72.

[3] Khalili Z, Moradi MH. Emotion recognition system using brain and peripheral signals: using correlation dimension to improve the results of EEG. In international joint conference on neural networks 2009 (pp. 1571-5). IEEE.

[4] Haag A, Goronzy S, Schaich P, Williams J. Emotion recognition using bio-sensors: first steps towards an automatic system. In tutorial and research workshop on affective dialogue systems 2004 (pp. 36-48). Springer, Berlin, Heidelberg.

[5] Ko KE, Yang HC, Sim KB. Emotion recognition using EEG signals with relative power values and bayesian network. International Journal of Control, Automation and Systems. 2009.

[6] Ringrose K. Law enforcement's pairing of facial recognition technology with body-worn cameras escalates privacy concerns. Online - Virginia Law Review. 2019; 105:57-66.
[7] Mythili S, Thiyagarajah K, Rajesh P, Shajin FH. Ideal position and size selection of unified power flow controllers (UPFCs) to upgrade the dynamic stability of systems: an antlion optimiser and invasive weed optimisation algorithm. HKIE Transactions. 2020; 27(1):25-37.

[8] Purshouse J, Campbell L. Privacy, crime control and police use of automated facial recognition technology. Criminal Law Review. 2019; 2019(3):188-204.

[9] Wilkinson S. Artificial intelligence, facial recognition technology and data privacy. Journal of Data Protection \& Privacy. 2020; 3(2):186-98.

[10] Baig Z, Shivakumar DS, Ananthapadmanabha DT. A multi-objective hybrid algorithm for planning electrical distribution system. International Journal of Electrical Engineering and Technology. 2020; 11(3):66-82.

[11] Leong B. Facial recognition and the future of privacy: i always feel like... somebody's watching me. Bulletin of the Atomic Scientists. 2019; 75(3):109-15.

[12] Martinez-martin N. What are important ethical implications of using facial recognition technology in health care?. AMA Journal of Ethics. 2019; 21(2): 180-7.

[13] Shajin FH, Rajesh P. Trusted secure geographic routing protocol: outsider attack detection in mobile ad hoc networks by adopting trusted secure geographic routing protocol. International Journal of Pervasive Computing and Communications. 2020.

[14] Hamann K, Smith R. Facial recognition technology. CRIM. JUST. 2019.

[15] Kostka G, Steinacker L, Meckel M. Between privacy and convenience: facial recognition technology in the eyes of citizens in China, Germany, the UK and the US. Germany, the UK and the US. 2020.

[16] Thota MK, Shajin FH, Rajesh P. Survey on software defect prediction techniques. International Journal of Applied Science and Engineering. 2020; 17(4):331-44.

[17] Conger K, Fausset R, Kovaleski SF. San Francisco bans facial recognition technology. The New York Times. 2019.

[18] Stark L, Stanhaus A, Anthony DL. "I don't want someone to watch me while i'm working": gendered views of facial recognition technology in workplace surveillance. Journal of the Association for Information Science and Technology. 2020; 71(9):1074-88.

[19] Morosan C. Disclosing facial images to create a consumer's profile: a privacy calculus perspective of hotel facial recognition systems. International Journal of Contemporary Hospitality Management. 2019; 31(8):3149-72.

[20] Kosinski M. Facial recognition technology can expose political orientation from naturalistic facial images. Scientific Reports. 2021.

[21] Varma S, Shinde M, Chavan SS. Analysis of PCA and LDA features for facial expression recognition using SVM and HMM classifiers. In techno-societal 2018 (pp. 109-19). Springer, Cham. 
[22] Kehri V, Ingle R, Patil S, Awale RN. Analysis of facial EMG signal for emotion recognition using wavelet packet transform and SVM. In machine intelligence and signal analysis 2019 (pp. 247-57). Springer, Singapore.

[23] Xu X, Zhang Y, Tang M, Gu H, Yan S, Yang J. Emotion recognition based on double tree complex wavelet transform and machine learning in internet of things. IEEE Access. 2019; 7:154114-20.

[24] Acharya D, Billimoria A, Srivastava N, Goel S, Bhardwaj A. Emotion recognition using fourier transform and genetic programming. Applied Acoustics. 2020.

[25] Zhang YD, Yang ZJ, Lu HM, Zhou XX, Phillips P, Liu QM, et al. Facial emotion recognition based on biorthogonal wavelet entropy, fuzzy support vector machine, and stratified cross validation. IEEE Access. 2016; 4:8375-85.

[26] Ayyavoo T, Suseela JJ. Illumination pre-processing method for face recognition using 2D DWT and CLAHE. IET Biometrics. 2018; 7(4):380-90.

[27] Krishna AH, Sri AB, Priyanka KY, Taran S, Bajaj V. Emotion classification using EEG signals based on tunable-Q wavelet transform. IET Science, Measurement \& Technology. 2019; 13(3):375-80.

[28] Meena HK, Sharma KK, Joshi SD. Facial expression recognition using the spectral graph wavelet. IET Signal Processing. 2019; 13(2):224-9.

[29] Ekman P, Friesen WV. Facial action coding systems. Consulting Psychologists Press; 1978.

[30] Ekman P. The argument and evidence about universals in facial expressions. Handbook of Social Psychophysiology. 1989.

[31] Sown M. A preliminary note on pattern recognition of facial emotional expression. In the international joint conferences on pattern recognition, 1978.

[32] Li C, Huang Y, Zhu L. Color texture image retrieval based on gaussian copula models of gabor wavelets. Pattern Recognition. 2017; 64:118-29.

[33] Wu T, Bartlett MS, Movellan JR. Facial expression recognition using gabor motion energy filters. In computer society conference on computer vision and pattern recognition-workshops 2010 (pp. 42-7). IEEE.

[34] Huang L, Xie F, Zhao J, Shen S, Guang W, Lu R. Human emotion recognition based on face and facial expression detection using deep belief network under complicated backgrounds. International Journal of Pattern Recognition and Artificial Intelligence. 2020; 34(14).

[35] Belhouchette K, Berkane M, Belhadef H. Interest emotion recognition approach using self-organising map and motion estimation. International Journal of Intelligent Systems Technologies and Applications. 2019; 18(5):494-508.

[36] Wei M. A novel face recognition in uncontrolled environment based on block 2D-CS-LBP features and deep residual network. International Journal of Intelligent Computing and Cybernetics. 2020; 13(2):207-21.
[37] Zhang QN. Improvement of face recognition technology based on intelligent image. In international conference on intelligent transportation, big data \& smart city 2019 (pp. 570-3). IEEE.

[38] Putri TT, Rachmawati E, Sthevanie F. Indonesian ethnicity recognition based on face image using uniform local binary pattern (ULBP) and color histogram. In international conference on informatics and computational sciences 2020 (pp. 1-5). IEEE.

[39] Li H. Application of image recognition based on grey relational analysis. Automatic Control and Computer Sciences. 2020; 54(4):371-7.

[40] Baker SA, Mohammed HH, Aldabagh HA. Improving face recognition by artificial neural network using principal component analysis. TELKOMNIKA. 2020; 18(6):3357-64.

[41] Shi D, Tang H. Face recognition algorithm based on self-adaptive blocking local binary pattern. Multimedia Tools and Applications. 2021; 80:23899921.

[42] Li Z, Cui LL. Three-dimensional image reconstruction method for human maxillofacial defect images based on block sample feature matching. International Journal of Engineering Systems Modelling and Simulation. 2019; 11(3):112-8.

[43] Nhat HT, Hoang VT. Feature fusion by using LBP, HOG, GIST descriptors and canonical correlation analysis for face recognition. In international conference on telecommunications 2019 (pp. 371-5). IEEE.

[44] Tran-trung K, Hoang VT. Hand gesture recognition under multi-view cameras using local image descriptors. In inventive computation and information technologies 2021 (pp. 299-304). Springer, Singapore.

[45] Qin Y, Sun L, Xu Y. Exploring of alternative representations of facial images for face recognition. International Journal of Machine Learning and Cybernetics. 2020; 11(10):2289-95.

[46] Mi JX, Zhu Q, Lu J. Principal component analysis based on block-norm minimization. Applied Intelligence. 2019; 49(6):2169-77.

[47] Liu J. Feature recognition method for similar key points of human face based on adaptive median filter. International Journal of Biometrics. 2021; 13(1):1-6.

[48] Mehta R, Satam S, Ansari M, Samantaray S. Realtime image processing: face recognition based automated attendance system in-built with "two-tier authentication" method. In international conference on data science and engineering 2020 (pp. 1-6). IEEE.

[49] Aiordachioaie D, Culea-florescu A, Pavel SM. On human faces thermal image processing for classification purposes. In 6th international symposium on electrical and electronics engineering 2019 (pp. 1-6). IEEE.

[50] Gnouma M, Ladjailia A, Ejbali R, Zaied M. Stacked sparse autoencoder and history of binary motion image for human activity recognition. Multimedia Tools and Applications. 2019; 78(2):2157-79.

[51] Majhi M, Pal AK. An image retrieval scheme based on block level hybrid DCT-SVD fused features. 
Multimedia Tools and Applications. 2021; 80(5):7271-312.

[52] Courville PL, Goodfellow A, Mirza IJ, Bengio Y. FER-2013 face database. Universit De Montreal: Montréal, QC, Canada. 2013.

[53] Lucey P, Cohn JF, Kanade T, Saragih J, Ambadar Z, Matthews I. The extended cohn-kanade dataset (ck+): a complete dataset for action unit and emotionspecified expression. In computer society conference on computer vision and pattern recognition-workshops 2010 (pp. 94-101). IEEE.

[54] Minaee S, Minaei M, Abdolrashidi A. Deep-emotion: facial expression recognition using attentional convolutional network. Sensors. 2021; 21(9):1-16.

[55] Aneja D, Colburn A, Faigin G, Shapiro L, Mones B. Modeling stylized character expressions via deep learning. In Asian conference on computer vision 2016 (pp. 136-53). Springer, Cham.

[56] Mehendale N. Facial emotion recognition using convolutional neural networks (FERC). SN Applied Sciences. 2020.

[57] Wang X, Chen X, Cao C. Human emotion recognition by optimally fusing facial expression and speech feature. Signal Processing: Image Communication. 2020.

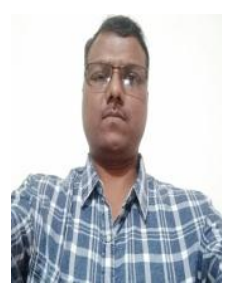

Dr. Pravin B Chopade received M.E in Electronics. Currently he is working as an Assistant Professor in Department of Electronics \& Telecommunication Engineering, Modern Education Society's College of Engineering, Pune, India for the past 17.6 years. His areas of interest are Signal and Image Processing. He is the member of ISTE and IETE. Email: drpbchopade@gmail.com

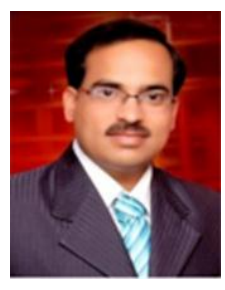

Prabhakar N. Kota received bachelor of engineering in Electronics and telecommunication in 2000. $\mathrm{He}$ received $\mathrm{M}$,Tech and $\mathrm{PhD}$ in Microwave. Currently he is Assistant Professor in Department of Electronics \& Telecommunication Engineering, Modern Education Society's College of Engineering, Pune, India for the past 17 years. His areas of interest are Wireless Communication, Digital Communication and Computer Network. He is the member of ISTE, IETE and IET.

Email: prabhakarnkota001@yahoo.com
Appendix I

\begin{tabular}{|c|c|c|}
\hline S. No. & Abbreviation & Description \\
\hline 1 & AAM & Active Appearance Model \\
\hline 2 & $\mathrm{AU}$ & Action Units \\
\hline 3 & BFA & Bimodal Fusion Algorithm \\
\hline 4 & $\mathrm{CK}+$ & Extended Cohn-Kanade \\
\hline 5 & $\mathrm{CNN}$ & $\begin{array}{ll}\text { Convolutional Neural } \\
\text { Networks }\end{array}$ \\
\hline 6 & DCT & Discrete Cosine Transform \\
\hline 7 & DFT & Discrete Fourier Transform \\
\hline 8 & DRN & Deep Residual Network \\
\hline 9 & DWT & Discrete Wavelet Transform \\
\hline 10 & FACS & Facial Action Coding System \\
\hline 11 & FD & Frequency Domain \\
\hline 12 & FFT & Fast Fourier Transform \\
\hline 13 & FER & $\begin{array}{ll}\text { Facial } & \text { Expression } \\
\text { Recognition } & \\
\end{array}$ \\
\hline 14 & FERG & $\begin{array}{l}\text { Facial Expression Research } \\
\text { Group Database }\end{array}$ \\
\hline 15 & GIST & Global Descriptors \\
\hline 16 & GMM & Gaussian Mixture Model \\
\hline 17 & GWT & Gabor Wavelet Transform \\
\hline 18 & HER & Human Emotion Recognition \\
\hline 19 & HER-BP-DWT & $\begin{array}{l}\text { Human Emotion Recognition } \\
\text { based on Block Patterns of } \\
\text { Image and Discrete Wavelet } \\
\text { Transform }\end{array}$ \\
\hline 20 & HER-BFA & $\begin{array}{l}\text { Human Emotion Recognition } \\
\text { using Bimodal Fusion } \\
\text { Algorithm }\end{array}$ \\
\hline 21 & HER-CNN & $\begin{array}{l}\text { Human Emotion Recognition } \\
\text { using Convolutional Neural } \\
\text { Networks }\end{array}$ \\
\hline 22 & HOG & $\begin{array}{l}\text { Histograms of Oriented } \\
\text { Gradients }\end{array}$ \\
\hline 23 & IoT & Internet of Things \\
\hline 24 & JAFFE & $\begin{array}{lll}\text { Japanese } & \text { Female } & \text { Facial } \\
\text { Expression } & & \\
\end{array}$ \\
\hline 25 & LBP & Local Binary Pattern \\
\hline 26 & $\mathrm{NN}$ & Neural Network \\
\hline 27 & PCA & $\begin{array}{l}\text { Traditional Principal } \\
\text { Component Analysis }\end{array}$ \\
\hline 28 & $\mathrm{R}-\mathrm{CNN}$ & $\begin{array}{l}\text { Region-based Convolutional } \\
\text { Neural Network }\end{array}$ \\
\hline 29 & SOM & Self-Organizing Map \\
\hline 30 & ULBP & Uniform Local Binary Pattern \\
\hline 31 & WT-BPCA & $\begin{array}{l}\text { Wavelet Transform on Block } \\
\text { PCA }\end{array}$ \\
\hline
\end{tabular}

